\title{
Pyroelectric Properties of PVDF:MWCNT Nanocomposite Film for Uncooled Infrared Detectors
}

\author{
Matthew E. Edwards, Ashok K. Batra, Ashwith K. Chilvery, Padmaja Guggilla, Michael Curley, \\ Mohan D. Aggarwal
}

Department of Physics, Chemistry and Mathematics, Alabama A\&M University, Normal, AL 35762, USA.

Email: ashok.batra@aamu.edu

Received October $12^{\text {th }}, 2012$; revised November $11^{\text {th }}, 2012$; accepted December $8^{\text {th }}, 2012$

\begin{abstract}
Pyroelectric multi-walled carbon nanotubes:polyvinylidene fluoride (PVDF:MWCT) composite films have been fabricated by the solution casting technique. The pyroelectric and dielectric properties of the composite films were examined for their use in uncooled infrared detectors. The properties measured include: 1) dielectric constants and 2) pyroelectric coefficient as a function of temperature. From the foregoing parameters, materials Figures-of-merit, for infrared detection and thermal-vidicons, were calculated. The results indicated Figures-of-merit of composite film were higher than pristine polyvinylidene fluoride films.
\end{abstract}

Keywords: Pyroelectric IR Detectors; Multi-Walled Carbon Nanotubes; Pyroelectric Properties

\section{Introduction}

Uncooled infrared (IR) detectors have rapidly advanced technologically over the past several years. In particular, IR detectors operating at room temperature are attractive to many civilian and military applications, such as night vision, detection of gas leakages, surveillance, fire rescue operations, manufacturing quality control, early fire detection and guidance, medical diagnostics, missile tracing and interception. There are two types of IR detectors: photon and thermal types. In general, photon types are preferred primarily due to their superior sensitivity and resolution. However, the photon sensors must be cryogenically cooled down during operation to obtain high sensitivity. Uncooled thermal sensors have attracted much attention because they operate at room temperature. These devices offer the advantage of low cost fabrication and a wide band of $8-14 \mu \mathrm{m}$ and beyond, operation coupled with a performance adequate for many applications, especially in consumer applications such as human detection, burglar alarms and door openers etc. The candidate materials for uncooled pyroelectric infrared detectors (PIR) are triglycine sulfate (TGS), lithium niobate (LN), lithium tantalate (LT), lead titanate (PT), lead zirconate titanate (PZT), polyvinylidene fluoride (PVDF), polyvinylidene fluoride-trifluoroethylene copolymer (P(VDF-TrFE)) and others [1-4]. PVDF, besides being flexible, has a number of attractive pyroelectric and piezoelectric properties with fast, dynamic response for use in touch/tactile sensors, and infrared detectors vidicon/imaging devices [5,6]. Carbon Nanotubes (CNTs) exhibit excellent mechanical and electrical properties of the order of $\sim 10^{3}-10^{4} \mathrm{~S} / \mathrm{cm}$. As the polymers present high strength, good flexibility, high transparency, easy processing, and low cost, combining polymers with CNTs would be promising for sensor materials [7]. Multi-walled nanotubes (MWCNT) consist of multiple layers of graphite, rolled in on themselves to form a tube shape.

In the present investigation, an effort was made to disperse multi-walled carbon nanotubes (MWCNTs) via ultrasonic processing in PVDF matrix to form 0 - 3 nanocomposite. The dielectric and pyroelectric properties of the resultant composite films were measured. After using forgoing parameters, to assess their use as pyroelectric infrared detectors, and as vidicons, various Figures-of-merit have been calculated and compared with pristine PVDF film.

\section{Experiment}

The 0 - 3 connectivity nanocomposite films were fabricated using the solution cast technique. The first step in the preparation of PVDF solution (Pmix) was to dissolve a suitable amount of PVDF in methyl-ethyl-ketone (MEK) at $60^{\circ} \mathrm{C}$. A requisite amount of MWCNT solution was then added to form nPMix, and this mixture was ultrasonically agitated/mechanically stirred, for several hours, to break-up the agglomerates and disperse MWCNT uniformly in the Pmix. The length and diameter of 
multi-walled carbon nanotubes were $10 \mu \mathrm{m}$ and $9-12$ $\mathrm{nm}$ (average) respectively. The obtained nPMix composite solution was kept in a suitable container for the solvent to evaporate. The films were annealed for $2-3$ hours in the air, at $110^{\circ} \mathrm{C}$ for the present case. A full-face silver electrode was deposited on the film (front and back faces); then the film was cut into $10 \mathrm{~mm} \times 10 \mathrm{~mm}$ sized element for testing. The electrode samples were poled at $60^{\circ} \mathrm{C}$ using $5 \mathrm{kV} / \mathrm{cm}$ for 2 hours. After the poling process, the samples were short circuited and annealed at $50^{\circ} \mathrm{C}$ for 2 hours. The flowchart of the fabrication processes is depicted in Figure 1.

The real $\left(\varepsilon^{\prime}\right)$ and imaginary $\left(\varepsilon^{\prime \prime}\right)$ parts of dielectric constant were calculated by measuring capacitance and dielectric loss. To measure the dynamic pyroelectric current, a direct measurement technique was used. The detailed measurement procedures of these parameters were described in our earlier publication [7]. The pyroelectric current $I_{p}$ was measured, and the pyroelectric coefficient $(p)$ was calculated using the relationship:

$$
p=\left(\frac{I_{p}}{A(d T / d t)}\right)
$$

where $A$ is the electrode area (identical areas for the opposite electrodes used in each sample) and $d T / d t$ is the rate of temperature change with time, which was kept to $1{ }^{\circ} \mathrm{C} /$ minute. Using the pyroelectric coefficient and di-

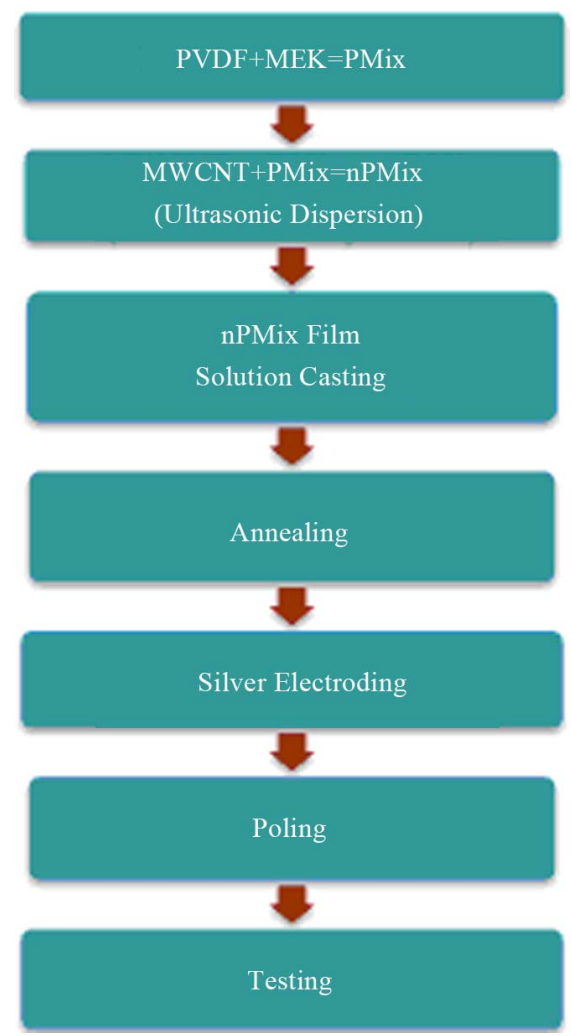

Figure 1. Summary of composite film fabrication process. electric constant, pyro-voltage (V) is calculated with $1^{\circ} \mathrm{C}$ change in temperature $(d T)$ as follows:

$$
Q(\text { charge generated })=p \cdot d T \cdot A \text { and } ?=Q / C
$$

The other important properties to look for in sensors and vidicons are low dielectric constant, low dielectric loss, high pyroelectric coefficient, and low specific heat. In this regard, important Figures-of-merit $(F M)$ [1-5] are:

$$
F M I=p / c^{\prime}
$$

for high current detectivity,

$$
F M_{v}=p / c^{\prime} \varepsilon^{\prime}
$$

for high voltage responsivity,

$$
F M d=p / c^{\prime} \sqrt{\left(\varepsilon^{\prime \prime}\right)}
$$

for high detectivity, and

$$
F M_{v i d}=p / c^{\prime} \sqrt{\left(\varepsilon^{\prime}\right)}
$$

for high vidicon responsivity, where $p$ is the pyroelectric coefficient, $c^{\prime}$ is the volume specific heat, $\varepsilon^{\prime}$ is the dielectric constant and $\varepsilon^{\prime \prime}$ is the imaginary part of dielectric constant (dielectric loss constant). In our calculations, it was assumed that the specific heat is the same in both PVDF and composite films so it is not used in calculations.

\section{Results and Discussions}

Figures $\mathbf{2}$ to $\mathbf{5}$ show the temperature dependence of the pyroelectric coefficient $p$, the dielectric constants $\varepsilon^{\prime}$, the dielectric loss constant $\varepsilon^{\prime \prime}$ of PVDF and nanocomposite films, respectively. As expected in ferroelectrics, these parameters increase with the increase in temperature. The variation of the pyroelectric coefficient of the films is depicted in Figures 2 and 3. The pyroelectric performance Figures-of-merit $\left(F M_{I}, F M_{v}, F M_{d}\right.$ and $\left.F M_{v i d}\right)$, which increase with the addition of MWCNT in the PVDF matrix, are presented in Figures 6 to 11. The resulting properties and Figures-of-merit of polymer and composite films are summarized in Table 1. It is evident from Table 1 that the pyroelectric coefficient and the Figuresof-merit of nanocomposite films are higher than pristine PVDF film poled under similar conditions. A theoretical model had been developed, which explains the effect of the matrix's electrical conductivities on the electrical properties and poling behaviors of the 0 - 3 ferroelectric composites [8,9]. Zhang et al. [8] developed a comprehensive analysis for the pyroelectric activity of $0-3$ ferroelectric composites, which includes additional contributions from the electrical conductivity of the constituents. According to Wong's model [9], the increment of conductivity of matrix increases the poling degree of the ceramic, and hence the pyroelectric properties are enhanced. The increment of the matrix's electrical conduc- 


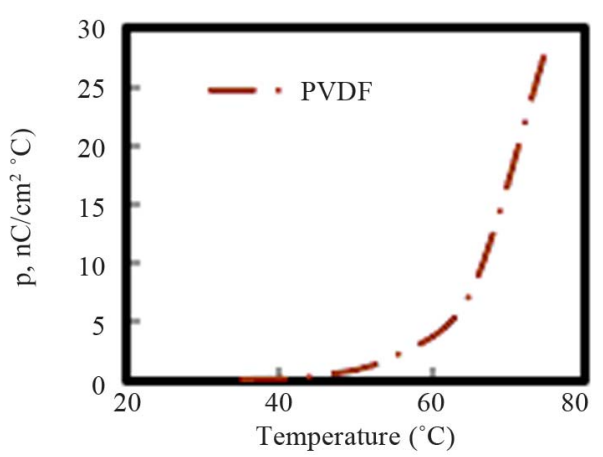

Figure 2. Pyroelectric coefficient vs temperature of PVDF film.

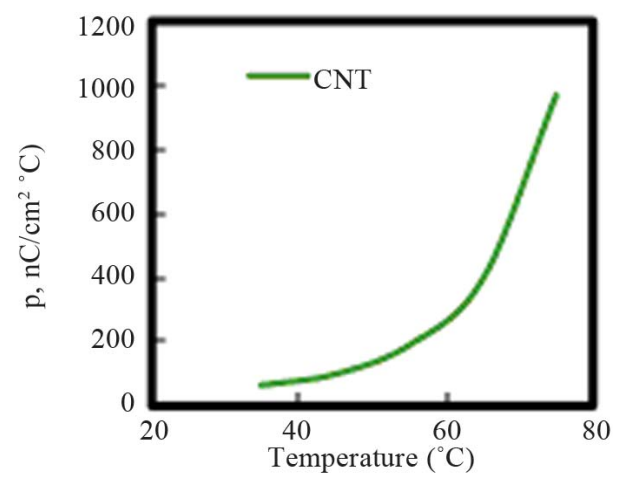

Figure 3. Pyroelectric Coefficient vs Temperature of PVDF/ MWCNT film.

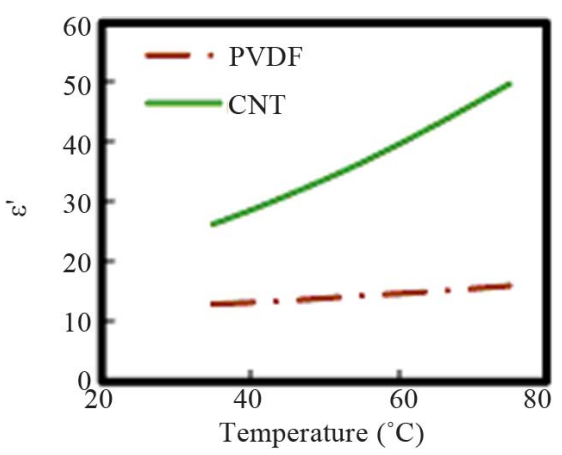

Figure 4. Real part of dielectric constant $\left(\varepsilon^{\prime}\right)$ vs temperature of PVDF and PVDF/MWCNT films.

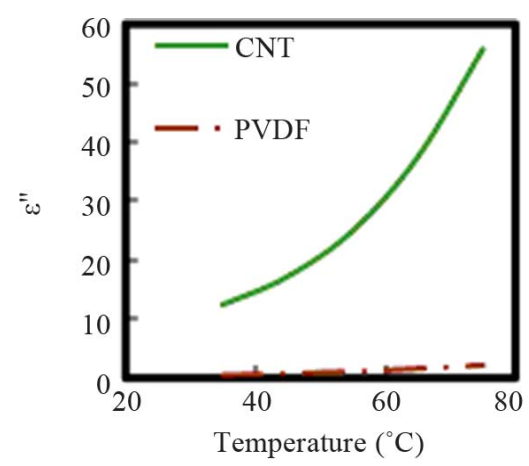

Figure 5. Imaginary part of dielectric constant $\left(\varepsilon^{\prime \prime}\right)$ vs temperature of PVDF and PVDF/MWCNT films.

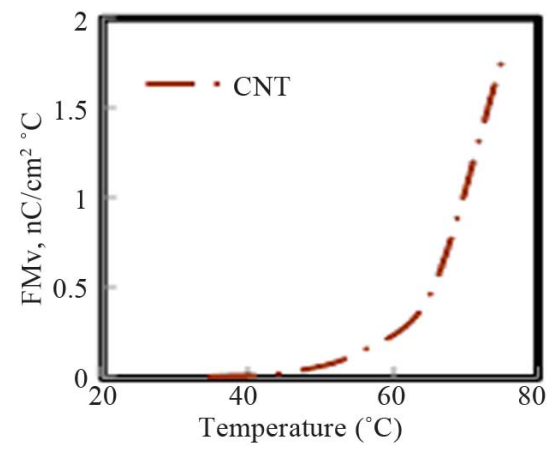

Figure 6. Figure-of-merit for responsivity vs temperature of PVDF film.

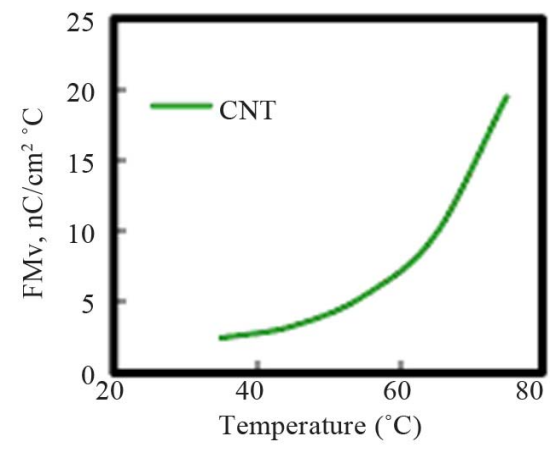

Figure 7. Figure-of-merit for responsivity vs temperature of PVDF/MWCNT film.

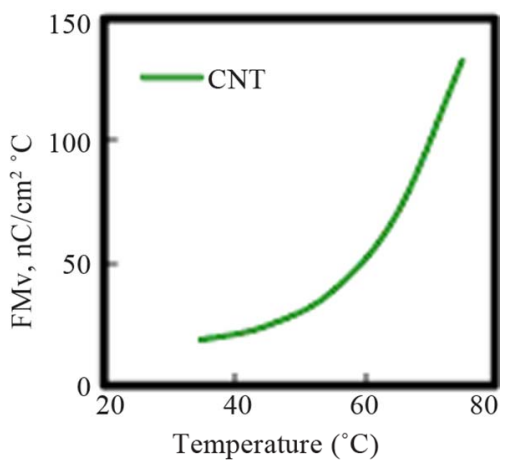

Figure 8. Figure-of-merit for detector applications vs temperature of PVDF/MWCNT film.

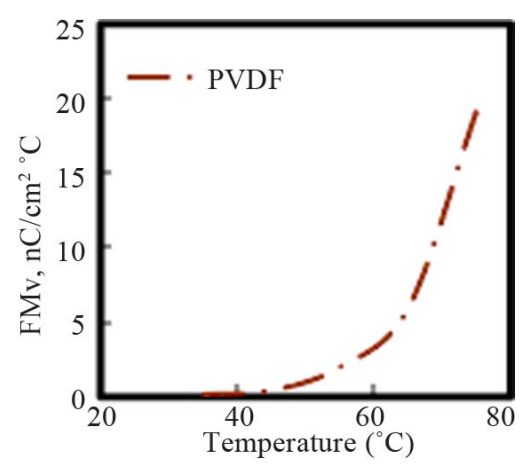

Figure 9. Figure-of-merit for detector applications vs temperature of PVDF film. 


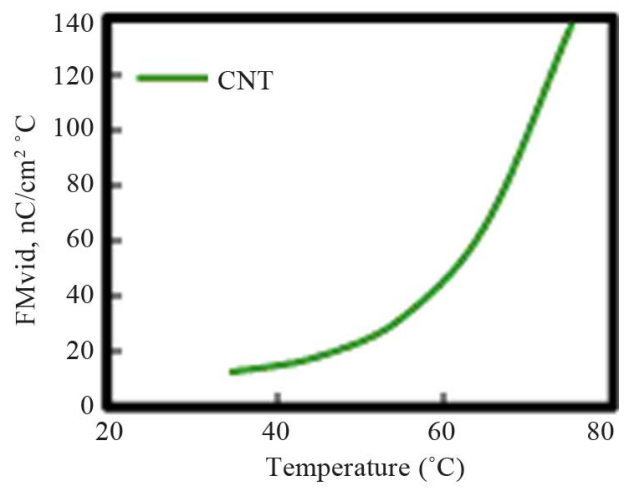

Figure 10. Figure-of-merit for vidicon application vs temperature of PVDF/MWCNT film.

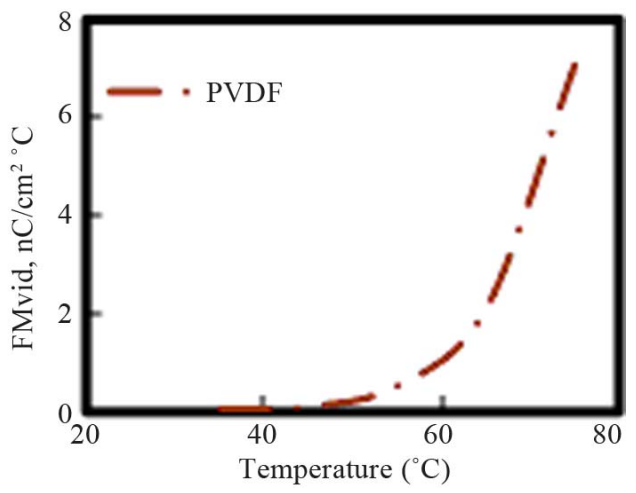

Figure 11. Figure-of-merit for vidicon application vs temperature of PVDF film.

Table 1. Dielectric, pyroelectric, and Figures-of-merit of PVDF and composites films.

\begin{tabular}{|c|c|c|c|c|c|c|c|}
\hline $\begin{array}{l}\text { Temp. } \\
\left({ }^{\circ} \mathrm{C}\right)\end{array}$ & $\begin{array}{l}\text { Pyroelectric Coeff. } \\
(\mathrm{p}) ; F_{I}\left(\mathrm{nC} / \mathrm{cm}^{2}{ }^{\circ} \mathrm{C}\right)\end{array}$ & $\begin{array}{l}\text { Dielectric } \\
\text { Cont. }\left(\varepsilon^{\prime}\right)\end{array}$ & $\begin{array}{l}\text { Dielectric } \\
\text { Loss }\left(\varepsilon^{\prime \prime}\right)\end{array}$ & Pyro-Voltage $(\mathrm{V})$ & $\begin{array}{c}F M_{V}=p / \varepsilon^{\prime} \\
\left(\mathrm{nC} / \mathrm{cm}^{2}{ }^{\circ} \mathrm{C}\right)\end{array}$ & $\begin{array}{c}F M_{v i d}=p /\left(\varepsilon^{\prime}\right)^{1 / 2} \\
\left(\mathrm{nC} / \mathrm{cm}^{2}{ }^{\circ} \mathrm{C}\right)\end{array}$ & $\begin{array}{c}F M_{d}=p /\left(\varepsilon^{\prime \prime}\right)^{1 / 2} \\
\left(\mathrm{nC} / \mathrm{cm}^{2}{ }^{\circ} \mathrm{C}\right)\end{array}$ \\
\hline \multicolumn{8}{|c|}{ PVDF Film } \\
\hline 35 & 0.12 & 12.92 & 0.47 & 3.16 & 0.01 & 0.032 & 0.17 \\
\hline 45 & 0.43 & 13.54 & 0.77 & 3.31 & 0.03 & 0.104 & 0.42 \\
\hline 55 & 2.09 & 14.37 & 1.10 & 3.51 & 0.13 & 0.523 & 1.98 \\
\hline 65 & 7.41 & 15.09 & 1.59 & 3.69 & 0.45 & 2.094 & 5.89 \\
\hline 75 & 28.3 & 16.02 & 2.16 & 3.92 & 1.76 & 7.067 & 19.16 \\
\hline \multicolumn{8}{|c|}{ PVDF:MWCNT Film } \\
\hline 35 & 63.9 & 26.17 & 12.33 & 10.19 & 2.45 & 12.468 & 18.21 \\
\hline 45 & 99.1 & 31.11 & 17.24 & 12.12 & 3.29 & 18.129 & 24.42 \\
\hline 55 & 189.07 & 36.57 & 24.98 & 14.24 & 5.43 & 32.195 & 38.42 \\
\hline 65 & 394.56 & 42.89 & 37.48 & 16.70 & 9.72 & 66.214 & 70.42 \\
\hline 75 & 968.41 & 49.76 & 55.72 & 19.38 & 19.47 & 137.282 & 132.82 \\
\hline
\end{tabular}

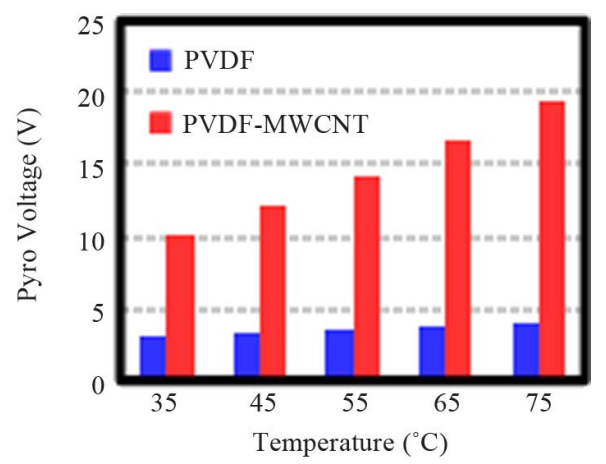

Figure 12. Comparison of pyrovoltage generated for PVDF and PVDF-MWCNT via $1^{\circ} \mathrm{C}$ increase in temperature.

tivity will increase the leakage current and the dielectric loss $\varepsilon^{\prime \prime}$, which will be harmful to the poling process. Our data are in agreement with the expected results [9].

Compared to pristine polymer films, composite film- system, PVDF:MWCNT, manifests increase in the pyroelectric constant, the dielectric constant, the dielectric loss with increasing temperature and as such have the tendency to provide a greater responsivity or detectivity, with application as an uncooled infrared sensor. It is evident from the Table 1 and the Figure 12 that the pyrovoltage generated is increasing with the increase in the temperature while it is being consistent in the pure PVDF. This increase in the investigated parameters in MWCNT composite films is attributed to the formation of a continuous network that allows efficient current to flow through the material.

\section{Conclusions}

The experimental results obtained of nanocomposite films (PVDF, PVDF-MWCNT films) can be summarized as follows: 
- Films of PVDF and PVDF: MWCNT have been fabricated using solution cast method. This technique is a very useful and inexpensive technique for manufacturing nanocomposite pyroelectric sensors because the films can be fabricated with less energy, time, and effort as compared to ceramic and single crystal fabrication.

- The films were characterized for their dielectric, and pyroelectric properties, in order to determine their usefulness in un-cooled infrared detector and vidicon, and tactile applications.

- The calculated pyroelectric Figures-of-merit (FMv, FMd and FMvid) of the nanocomposite films were found to be higher than pure PVDF films.

- Results show that the pyro-voltage generated by the addition of the MWCNTs is increasing with the increase in temperature.

- Based on the preliminary results obtained, PVDF: MWCNT films are attractive for use in uncooled thermal sensing elements especially in applications where flexible and curved-surface based sensors are required. Further work is in progress to ascertain the mechanisms for enhancement of dielectric and pyroelectric properties of nanocomposites films.

\section{Acknowledgements}

This work is partially supported by NSF-EPSCoR R-II-3, grant \# EPS-1158862; DHS grant 2010-ST-062-000034; and the Evans Allen research grant. Special thanks to Dr. Kunal Grover, MD., Department of Internal Medicine, Henry Ford Hospital, Detroit, MI 48202, for his invaluable comments and suggestions.

\section{REFERENCES}

[1] A. K. Batra, M. D. Aggarwal, M. E. Edwards and A. Bhalla, "Present Status of Polymer: Ceramic Composites for Pyroelectric Infrared Detectors," Ferroelectrics, Vol. 366, No. 1, 2008, pp. 84-121. doi:10.1080/00150190802363207

[2] A. K. Batra, M. A. Alim, J. R. Currie and M. D. Aggarwal, "The Electrical Response of the Modified Lead Titanate Based Thick-Films," Physica B: Condensed Matter, Vol. 404, No. 14-15, 2009, pp. 1905-1911. doi:10.1016/j.physb.2009.02.024

[3] M. D. Aggarwal, A. K. Batra, P. Guggilla, M. E. Edwards, B. Penn and J. R. Currie, "Pyroelectric Materials for Uncooled Infrared Detectors," NASA Technical Memorandum-NASA/TM, 2010, pp. 1-71, Article ID: 2164373.

[4] M. E. Edwards, A. K. Batra, A. K. Chilvery, P. Guggilla and M. D. Aggarwal, "Characterization of Polymeric Composite Films with MWCNT and Ag Nanoparticles," Proceedings of SPIE, San Diego, 12 August 2012.

[5] L. Seminara, M. Capurro, P. Cirillo, G. Cannata and M. Valle, "Electromechanical Characterization of Piezoelectric PVDF Polymer Films for Tactile Sensors in Robotics Applications," Sensors and Actuators A: Physical, Vol. 169, No. 1, 2011, pp. 49-58. doi:10.1016/j.sna.2011.05.004

[6] I. Graz, K. Markus, B.-G. Simona, et al., "Flexible Active-Matrix Cells with Selectively Poled Bifunctional Polymer Ceramics Nanocomposites for Pressure and Temperature Sensing Skins," Journal of Applied Physics, Vol. 106, No. 3, 2009, Article ID: 034503. doi:10.1063/1.3191677

[7] A. K. Batra, J. Corda, P. Guggilla, M. D. Aggarwal and M. E. Edwards, "A Electrical Properties of Silver Nanoparticles Reinforced $\mathrm{LiTaO}_{3}: \mathrm{P}(\mathrm{VDF}-\mathrm{TrFE})$ Composite Films," Proceedings of SPIE, San Diego, 12 August 2009.

[8] D.-M. Zhang, N. Wei, F.-X. Yang, X.-Y. Han, et al., "A New Comprehensive Model for the Pyroelectric Property of 0-3 Ferroelectric Composites," Journal of Physics D: Applied Physics, Vol. 39, 2006, pp. 1963-1969. doi:10.1088/0022-3727/39/9/037

[9] C. K. Wong and F. G. Shin, "Electrical Conductivity Enhanced Dielectric and Piezoelectric Properties of Ferroelectric 0-3 Composites," Journal of Physics D: Applied Physics, Vol. 97, No. 6, 2005, Article ID: 064111. doi:10.1063/1.1862317 